\section{Cognitive Control Therapy as a Depression Treatment: A Review of the Literature}

\section{Abstract}

Recent investigations have explored the link between depression and executive function (the ability to process and organise information in order to achieve a goal). The specific aspect of executive function that is linked to depression is executive control, which includes the ability to regulate cognitive processes such as emotion. Research has found that subjects with greater levels of executive control are more likely to be able to control their reactions to stressful events and are less likely to develop depressive symptoms. As a result of these findings, cognitive control training has been suggested as a possible new treatment for depression. Further research in this field would be highly beneficial for the development of new and effective treatments for depression.

Keywords: Depression; Executive function; Therapeutics; Cognitive; Mental health

Received: May 12, 2017; Accepted: May 25, 2017; Published: May 31, 2017

\begin{abstract}
Alicia JS Davis
Soka University of America, University Drive, Aliso Viejo, CA 92656, USA
\end{abstract}

\section{Corresponding author: Alicia JS Davis}

” aldavis@soka.edu

Soka University of America, University Drive, Aliso Viejo, CA 92656, USA.

Tel: $+1(949) 480-4590$

Citation: Davis AJS. Cognitive Control Therapy as a Depression Treatment: A Review of the Literature. Acta Psychopathol. 2017, 3:3.

\section{Introduction}

Depression is a complicated mental illness that affects more than $6.7 \%$ of American adults [1-5]. High depression rates have increased research on effective treatments [5-7]. A Major Depressive Disorder (MDD) diagnosis is based on presentation of 5 or more symptoms, including: (1) Depressed mood for most of the day and nearly every day, (2) Diminished interest or pleasure in activities, (3) Significant weight fluctuations, (4) Insomnia, and (5) Feelings of worthlessness [8]. In most people, depression can be triggered by stressful events such as death, job loss, or divorce, in addition to poor diet, exercise, and sleep or biologically caused imbalances in neurotransmitters [5]. Although psychologically healthy people are able to recover from a depressive episode, others develop a prolonged condition called dysthymia or Persistent Depressive Disorder (PDD). This disorder is characterised by the symptoms above lasting more than 2 years for adults and more than 1 year for children [8].

Researchers have formed a consensus that depression rates have increased significantly over time [6,7,9]. Depression was first studied in the 1900s; however, many problems, including recall bias and inconsistent findings, plagued preliminary research [9]. Discrepancies in how the condition was diagnosed throughout history also caused problems in comparing and interpreting data. For this reason, at best we can only estimate depression rates in the past, but there is evidence that over the last 40 years these rates have increased $[6,7,9]$. One study found that prevalence of depression in American adults increased from 3.33\% to $7.06 \%$ between 1991-92 and 2001-02 [9]. Other research has also discovered an increase in suicide rates between 1999 and 2013, linked to mental health problems [7]. Because of these rising statistics, psychologists have aggressively pursued causes and treatments.

Current treatments for MDD and dysthymia include medication and psychotherapies such as Cognitive Behavioural Therapies (CBT) [10]. Although CBT has been employed extensively in the past, recent research has determined that the successfulness of the therapy declines after time [11]. The usefulness of medications such as selective Serotonin Reuptake Inhibitors (SSRIs) has also been recently questioned. In studies conducted where both an SSRI and a placebo were administered to patients, differences in effectiveness were not extremely significant [12]. Therefore, further research towards an efficacious treatment of depressive symptoms is necessary.

\section{Links between Cognitive Control and Depression}

Recent investigations have studied the genetic and physical make-up of the brain which has resulted in a better understanding of executive function-the ability to process and organise information $[1,3,13]$. Executive function encompasses mental processes that include working memory, emotion identification, and planning, as well as cognitive control processes such as 
inhibition, reactivity regulation, and selective attention [14]. For example, Maalouf et al. [2], Quinn and Joormann [4] and Kertz et al. [14] explored the link between decreased executive function and cognitive control capabilities, and probability of exhibiting depressive symptoms. Through comparing executive function levels and depression symptoms of patients, the researchers investigated how a person's unique executive function may cause either susceptibility or resistance to depressive symptoms. Research has found that subjects who have greater levels of executive control are able to control their reactions to stressful events and experience less negative affectivity. Subjects with higher levels of executive control are therefore less likely to exhibit depressive symptoms.

Maalouf et al. [2] studied the relationship between executive function and depression. The researchers administered three tasks that measured the effectiveness of executive function of participants with depression compared to control participants. Higher levels of negative affectivity and sequentially symptoms of MDD were correlated with executive function deficits in patients when compared to psychologically healthy subjects and subjects in remission $[15,16]$. Maalouf et al. [2] demonstrated that executive function has a two-fold relationship with depression in that executive function may increase depressive symptoms and executive control levels may be decreased by symptoms.

Kertz et al. [14] studied cognitive control capacity and its link to depression in later school years of 188 children aged 3-5. The investigators conducted baseline tests to determine the children's executive function levels and mental health, with subsequent follow-up tests over a 7-year period to track their development. The study found that cognitive control deficits were highly correlated with increased rates of anxiety and depression in children over the 7-year period. This finding suggests that executive function potentially relates to effective management of depressive symptoms in later childhood and into adulthood.

Quinn and Joormann [4] studied the correlation between executive function and ability to control emotions, investigating whether stress-related decline in executive function leads to increased depression symptoms. The researchers first determined a baseline of the participants' depression symptoms and prior stressful life events using the Beck Depression Inventory and Negative Life Events Questionnaire, both self-report questionnaires. The researchers then assessed participants' executive control using the n-back task which measures how fast participants were able to correctly identify and match words that were previously presented to them [4].

After the baseline depression, stress, and executive control levels were found, participants were exposed to a stressful situation that required them to prepare and give a speech. Following this, the participants were reassessed to investigate any changes in executive control, stress levels, and depression levels. The participants experienced a decline in executive control after the stressful experience, leading to an increase in depressive symptoms. Quinn and Joormann [4] concluded that a negative affect response to stressful situations can be attributed to decreased emotional control. The significance of the results is that executive control decreases following stressful situations, which manifests in depressive symptoms. This data corroborates Maalouf et al. [2] results.

\section{Research on Cognitive Control Therapy}

Researchers have been able to successfully identify that executive control plays a major role in depression. Recently, research has been conducted as to whether an increase in executive control would decrease depression symptoms. Prominent studies including those of lacoviello et al. [17], Hoorelbeke and Koster [15], and Siegle et al. [18] have investigated benefits of cognitive control training as a therapy for depression.

lacoviello et al. [17] administered cognitive-emotional control training, consisting of identifying and sequencing emotions of people, to a treatment group of 11 participants, and basic control training with neutral stimulus to a control group of 10 participants. The cognitive-emotional control training targeted improvement in working memory and emotion identification, two aspects of executive function. The researchers tracked the initial executive function and depression levels of the participants, and tracked changes over 4 weeks. Patients who were exposed to cognitiveemotional control training saw improvements in their depression and executive function levels, and a majority achieved a clinical response (equal to or more than $50 \%$ reduction in symptoms), despite the short period of training.

Hoorelbeke and Koster [15] conducted a similar but more extensive study investigating the efficacy of cognitive control training in decreasing the likelihood of depression symptoms continuing or reoccurring in patients in remission. Researchers selected a treatment group of 34 subjects who received the cognitive control training task named the Paced Auditory Serial Addition Task (PASAT), consisting of listening to a continuous stream of numbers and responding with the sum of the last two numbers. The cognitive control training task was aimed at improving selective attention, working memory and aspects of executive control. A control group of 34 participants received a low cognitive load task which did not aim at improving executive function. Participants were subjected to 10 sessions of training over a period of 14 days. Researchers assessed whether depressive rumination and depressive symptomology were improved immediately and twelve weeks after training. The results showed that both depressive rumination and depressive symptomology were lowered after cognitive training in all assessments, suggesting a potential clinical application for cognitive control training in treating depression [14]. Although this study employed only 68 participants, the results are promising in the face of rising US depression rates $[6,7,9]$.

Siegle et al. [18] also investigated cognitive control training as a treatment for depression. The researchers introduced the idea of combining existing depression therapies with control training. Executive function and increase in control over reactivity was said to positively augment the effects of antidepressants that already aim at effective reactivity regulation [18]. The experiment 
consisted of a treatment group of 27 participants receiving six sessions of a selective attention training exercise, the PASAT as a cognitive control task aiming to improve working memory and executive control, as well as treatment as usual. A control group of 26 participants only received treatment as usual. Usual treatments included use of antidepressants and group therapy. Initial executive function, rumination and depression levels were measured and monitored a year before the study, throughout the study, and a year after the study. The study found that patients who were exposed to cognitive control training had lower severity of depression symptoms and lower levels of rumination, with lasting benefits throughout the year post treatment. Cognitive control training was also found to increase effectiveness of executive function.

\section{Conclusion}

The issue of growth in depression rates needs to be countered with the innovation of new treatments. Research has demonstrated that decreased cognitive control and executive function can cause and also be an effect of depression $[2,4,15]$. Continued research into the benefits of cognitive control training is necessary to determine its efficacy as a viable treatment for depression and depressive symptoms. Using cognitive control training therapies accompanied by CBT and pharmaceuticals gives patients another option, as these existing therapies are not always successful solitarily [11,12]. With greater funding and more resources dedicated to developing cognitive control therapies, it is possible to further develop an innovative and successful treatment that will benefit a large portion of the American population. 


\section{References}

1 Barrasso-Catanzaro C, Eslinger PJ (2016) Neurobiological bases of executive function and social-emotional development: typical and atypical brain changes. Fam Relat: Interdiscipl J Appl Fam Stud 65: 108-119.

2 Maalouf FT, Klein C, Clark L, Sahakian BJ, LaBarbara EJ, et al. (2010) Impaired sustained attention and executive dysfunction: Bipolar disorder versus depression-specific markers of affective disorders. Neuropsychologia 48: 1862-1868.

3 Cybele RC, Blair C (2016) Neuroscientific insights: attention, working memory, and inhibitory control. Future Child 26: 95-118.

4 Quinn ME, Joormann J (2015) Control when it counts: Change in executive control under stress predicts depression symptoms. Emotion 15: 522-530.

5 https://www.nimh.nih.gov/health/statistics/prevalence/majordepression-among-adults.shtml

6 Hidaka BH (2012) Depression as a disease of modernity: explanations for increasing prevalence. J Affect Disord 140: 205-214.

7 Case A, Deaton A (2015) Rising morbidity and mortality in midlife among white non-Hispanic Americans in the $21^{\text {st }}$ century. Proc Natl Acad Sci USA 112: 15078-15083.

8 American Psychiatric Association (APA) (2013) Diagnostic and statistical manual of mental disorders: DSM-5. Washington, DC: American Psychiatric Publishing.

9 Compton WM, Conway KP, Stinson FS, Grant BF (2006) Changes in the prevalence of major depression and comorbid substance use disorders in the United States between 1991-1992 and 2001-2002. Am J Psychiatry 12: 2141-2147.

10 http://www.mayoclinic.org/diseases-conditions/depression/
11 Johnsen TJ, Friborg O (2015) The effects of cognitive behavioral therapy as an anti-depressive treatment is falling: A meta-analysis. Psychol Bull 141: 747-768.

12 Lawrence HR, Nangle DW, Schwartz-Mette RA, Erdley CA (2017) Medication for child and adolescent depression: questions, answers, clarifications, and caveats. Practice Innovations 2: 39-53.

13 Mous SE, Schoemaker NK, Blanken LE, Thijssen S, van der Ende J, et al. (2017) The association of gender, age, and intelligence with neuropsychological functioning in young typically developing children: the generation R study. Appl Neuropsychol Child 6: 22-40.

14 Kertz SJ, Belden AC, Tillman R, Luby J (2016) Cognitive control deficits in shifting and inhibition in preschool age children are associated with increased depression and anxiety over 7.5 years of development. J Abnorm Child Psychol 44: 1185-1196.

15 Hoorelbeke K, Koster EW (2017) Internet-delivered cognitive control training as a preventive intervention for remitted depressed patients: evidence from a double-blind randomized controlled trial study. J Consult Clin Psychol 85: 135-146.

16 Bridgett DJ, Oddi KB, Laake LM, Murdock KW, Bachmann MN (2013) Integrating and differentiating aspects of self-regulation: effortful control, executive functioning, and links to negative affectivity. Emotion 13: 47-63.

17 lacoviello BM, Wu G, Alvarez E, Huryk K, Collins KA, et al. (2014) Cognitive-emotional training as an intervention for major depressive disorder. Depress Anxiety 31: 699-706.

18 Siegle GJ, Price RB, Jones NP, Ghinassi F, Painter T, et al. (2014) You gotta work at it: Pupillary indices of task focus are prognostic for response to a neurocognitive intervention for rumination in depression. Clin Psychol Sci 2: 455-471. 